\title{
ADSORÇÃO DE CROMO (VI) EM LEITO FIXO UTILIZANDO PARTÍCULAS DE AREIA RECOBERTAS COM QUITOSANA
}

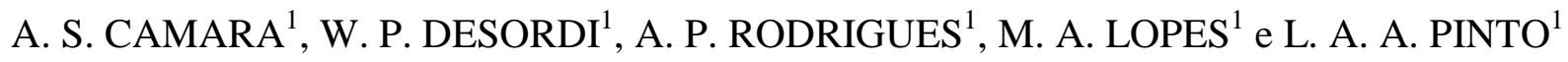 \\ ${ }^{1}$ Universidade Federal do Rio Grande, Escola de Química e Alimentos \\ E-mail para contato: alisson_camara@hotmail.com
}

\begin{abstract}
RESUMO - O objetivo desse trabalho foi avaliar o desempenho de partículas de areia recobertas com quitosana pelo método de recobrimento por imersão (dip-coating), como recheio de leito fixo, na adsorção de cromo (VI) de solução aquosa. Foram estudados os efeitos da vazão de alimentação $(2,5 ; 5,0$ e $10 \mathrm{~mL} / \mathrm{min})$ e do tamanho das partículas de areia recobertas com quitosana $(0,4 ; 0,7$ e $1 \mathrm{~mm})$ no processo de adsorção no leito fixo. $\mathrm{O}$ estudo mostrou que a diminuição da vazão melhorou o desempenho no processo de adsorção no leito, aumentando a capacidade total de adsorção $\left(q_{t}\right)$ de 6,02 para $15,6 \mathrm{mg} / \mathrm{g}$ e aumentando a quantidade de cromo (VI) retida no leito até sua ruptura $\left(q_{u}\right)$ de 3,8 para 14,8 $\mathrm{mg} / \mathrm{g}$. O diâmetro das partículas de areia recobertas com quitosana não teve influência significativa $(\mathrm{p}<0,05)$ na adsorção de cromo $(\mathrm{VI})$ dentro da faixa estudada.
\end{abstract}

\section{INTRODUÇÃO}

Os metais surgem nas águas naturais devido ao lançamento de efluentes provenientes de atividades industriais, tais como, mineração, galvanoplastia, indústrias de ferro, lavanderias, indústrias de petróleo entre outras. Os compostos de cromo destacam-se na indústria mundial, e estima-se que a sua produção é na ordem de 107 toneladas por ano e desse total, 60 a $70 \%$ vem da produção de ligas e em torno de $15 \%$ em processos químicos (Albadarin et al., 2012).

Diversos métodos incluindo precipitação química, eletrodeposição, trocas iônicas, separações por membranas e adsorção tem sido usados no tratamento de efluentes contendo metais, incluído o cromo. Os métodos convencionais são economicamente desfavoráveis e/ou tecnicamente complicados no tratamento de efluentes contendo baixas concentrações de metais. Nessa situação, os métodos tradicionais já não são vantajosos e a adsorção apresenta grande potencial, por ser um método eficiente e relativamente fácil de ser aplicado (Guibal, 2004). Os efluentes contendo cromo são muito difíceis de tratar, uma vez que os íons são altamente solúveis, dificultando o tratamento de efluentes em baixas concentrações de cromo (Albadarin $e t$ al., 2012). Comercialmente o carvão ativado é o adsorvente mais utilizado na remoção de contaminantes, mas existem diversos estudos com adsorventes alternativos e nesse cenário a quitosana ganha destaque, sendo utilizada na adsorção de diversos metais, tais como, cobre (Osifo et al., 2008), platina e paládio (Wang et al., 2011) e cromo (Aydin e Aksoy, 2009).

A utilização da quitosana em sistemas operando em batelada é amplamente estudada na 


\section{9 a 22 de outubro de 2014 \\ Florianópolis/SC}

literatura em ensaios de adsorção, pois são simples de serem aplicados em laboratório e fornecem informações importantes sobre o adsorvente frente ao adsorbato. Entretanto, em operações industriais a maior parte dos processos de adsorção é em leito fixo (Gerente et al., 2007). Porém, a utilização de quitosana diretamente em coluna de leito fixo não é aconselhável devido às características da partícula, e a imobilização da quitosana em uma superfície é uma alternativa para contornar esse problema (Guibal, 2004; Futalan et at., 2010).

Nesse contexto, o objetivo deste trabalho foi avaliar a influência do tamanho das partículas de areia recobertas com quitosana pelo método de recobrimento por imersão (dip-coating), como recheio de leito fixo na adsorção de cromo (VI) de solução aquosa, e a influência da vazão de alimentação no desempenho do leito fixo.

\section{MATERIAL E MÉTODOS}

\subsection{Recobrimento das Partículas de Areia (dip-coating)}

A quitosana foi obtida a partir de resíduos de camarão (Penaeus brasiliensis) fornecidos por uma indústria pesqueira da cidade do Rio Grande/RS. Os resíduos foram desmineralizados, desproteinizados e desodorizados para a obtenção da quitina. A quitina foi desacetilada utilizando solução alcalina concentrada (Weska et al., 2007), e a quitosana obtida foi purificada e seca em leito de jorro (Dotto et al., 2011). A quitosana foi caracterizada quanto ao grau de desacetilação (GD), massa molar e diâmetro de partícula, sendo GD determinado pelo método de titulação potenciométrica (Jiang et al., 2003), a massa molar determinada pelo método viscosimétrico (Moura et al., 2011) e o diâmetro de partícula pela definição de Sauter (Foust et al., 1980).

A areia da classe quartzítica foi obtida no comércio de Rio Grande - RS e foi submetida a moagem para homogeneização e após, submetida ao processo de peneiramento, utilizado um sistema de peneiras padronizados da série Tyler, obtendo-se frações com diâmetro médio equivalente a média entre a fração passante e a fração retida. Foram separadas as frações com diâmetro médio (1,0;0,7 e 0,4 mm) para o processo de recobrimento pela técnica dip-coating.

As partículas de areia foram recobertas utilizando a técnica dip-coating (Vijaya et al., 2008). O processo de recobrimento geralmente é composto por três etapas: (a) limpeza; (b) recobrimento; (c) cura. As partículas de areia passaram pela etapa de limpeza, sendo imersas em solução de ácido acético $3 \%(\mathrm{v} / \mathrm{v})$ por $6 \mathrm{~h}$ à $25 \pm 2{ }^{\circ} \mathrm{C}$, e na sequência passaram pela etapa de recobrimento, ficando imersas por $12 \mathrm{~h}$ em solução de quitosana $0,5 \%(\mathrm{p} / \mathrm{v})$ à $25 \pm 2{ }^{\circ} \mathrm{C}$. Após, foi realizada a etapa de cura física para a fixação do recobrimento, submetendo as partículas à temperatura de $45^{\circ} \mathrm{C}$ por $24 \mathrm{~h}$ (Laporte et al., 1997).

\subsection{Caracterização das Partículas Recobertas com Quitosana}

A quantidade de quitosana aderida à superfície das partículas foi determinada pela concentração de quitosana remanescente na solução de recobrimento, através do método 
colorimétrico (Muzzarelli, 1998), utilizando um espectrômetro na região do visível (Quimis, Q108 DRM, Brasil). A análise da superfície das partículas antes e após o recobrimento foi realizada através de microscopia eletrônica de varredura (MEV), com um microscópio eletrônico (Jeol, JSM 6060, Japão). As amostras foram metalizadas com carbono e foram utilizadas acelerações de voltagem de $10 \mathrm{kV}$ e faixas de magnificação variando de 50 a 1000 vezes.

\subsection{Ensaio Dinâmico de Adsorção}

Nos ensaios de adsorção em leito fixo, foi utilizada uma coluna cilíndrica de acrílico com diâmetro interno de $3,4 \mathrm{~cm}$, altura de $30 \mathrm{~cm}$, acoplada a uma bomba peristáltica (MasterFlex, 07553-75, Canadá). O efeito do tamanho da partícula de areia $(0,4 ; 0,7$ e 1,0 mm) e da vazão $(2,5 ; 5,0$ e $10,0 \mathrm{~mL} / \mathrm{mim})$ da solução aquosa de cromo (VI) foi avaliado nos experimentos a temperatura ambiente $\left(25 \pm 2{ }^{\circ} \mathrm{C}\right)$. A solução estoque $(1,0 \mathrm{~g} / \mathrm{L})$ de cromo (VI) foi preparada com dicromato de potássio $\left(\left(\mathrm{K}_{2} \mathrm{Cr}_{2} \mathrm{O}_{7}\right)\right.$ e o pH da solução foi ajustado em 3,0 com solução tampão (fosfato de sódio dibásico /ácido cítrico) $0,1 \mathrm{~mol} / \mathrm{L}$. A solução aquosa de cromo (VI) foi bombeada no sentido ascendente e amostras foram coletas no topo da coluna em intervalos de tempo regulares até a saturação do leito. A concentração remanescente na solução foi determinada por espectrofotometria (Quimis Q108 DRM, Brasil) a $540 \mathrm{~nm}$ utilizando o método da difenilcarbazida. Todos os experimentos foram realizados em réplica $(n=3)$.

Para a avaliação da influência do tamanho da partícula, trabalhou-se com a vazão de 5 mL/mim, pH 3,0 e a concentração inicial da solução de cromo (VI) em 100 mg/L. A massa de areia recoberta com quitosana foi de $350 \mathrm{~g}$ para todos os tamanhos de partícula, sendo a proporção de quitosana aderida na superfície da areia de 1,4 mg de quitosana por grama de areia. No estudo do efeito da vazão no desempenho do leito fixo, fixou-se a massa de areia no leito em 350 g, diâmetro de partícula 1,0 mm, pH 3,0 e a concentração inicial da solução em 100 mg/L.

A quantidade de íon metálico retida no leito $\left(q_{t}\right)$ até a saturação foi obtida por balanço de massa na coluna, usando os dados de saturação da mesma, a partir das curvas de ruptura, sendo que a área abaixo da curva $\left(1-C / C_{0}\right)$ é proporcional à quantidade de íon metálico retido, essa quantidade foi calculada pela Equação 1. A quantidade de íon retida no leito até o ponto de ruptura, que corresponde à capacidade útil da coluna $\left(q_{u}\right)$, foi calculada pela Equação 2.

$$
\begin{aligned}
& q_{t}=\frac{C_{0} V}{1000 m} \int_{0}^{t}\left(\frac{\left.C\right|_{Z=L}}{C_{0}}\right) d t \\
& q_{u}=\frac{C_{0} V}{1000 m} \int_{0}^{t b}\left(\frac{\left.C\right|_{Z=L}}{C_{0}}\right) d t
\end{aligned}
$$

sendo $q_{t}$ a capacidade total de adsorção do íon metálico $(\mathrm{mg} / \mathrm{g}), q_{u}$ a capacidade de adsorção do íon metálico até a ruptura do leito $(\mathrm{mg} / \mathrm{g}), m$ a massa seca de quitosana $(\mathrm{g}), V$ a vazão 
volumétrica da solução $\left(\mathrm{cm}^{3} / \mathrm{min}\right), C_{Z=L}$ a concentração do íon metálico na saída da coluna $(\mathrm{mg} / \mathrm{L}), C_{0}$ a concentração inicial do íon metálico $(\mathrm{mg} / \mathrm{L}), t_{b}$ o tempo até o ponto de ruptura (min) e $t$ o tempo ( $\mathrm{min})$.

Para o cálculo da do comprimento da zona de transferência de massa (ZTM) (Geankoplis, 1993) apresenta um método simplificado. Este pode ser obtida pela Equação 3, onde $H$ é a altura da coluna de adsorção.

$$
Z T M=H\left(1-\frac{q_{u}}{q_{t}}\right)
$$

\section{RESULTADOS E DISCUSSÃO}

A quitosana utilizada nos experimentos apresentou massa molar de $150 \pm 3 \mathrm{kDa}$, grau de desacetilação de $85 \pm 1 \%$ e diâmetro de partícula de $72 \pm 3 \mu \mathrm{m}$.

\subsection{Imagens de Microscopia Eletrônica de Varredura da Quitosana}

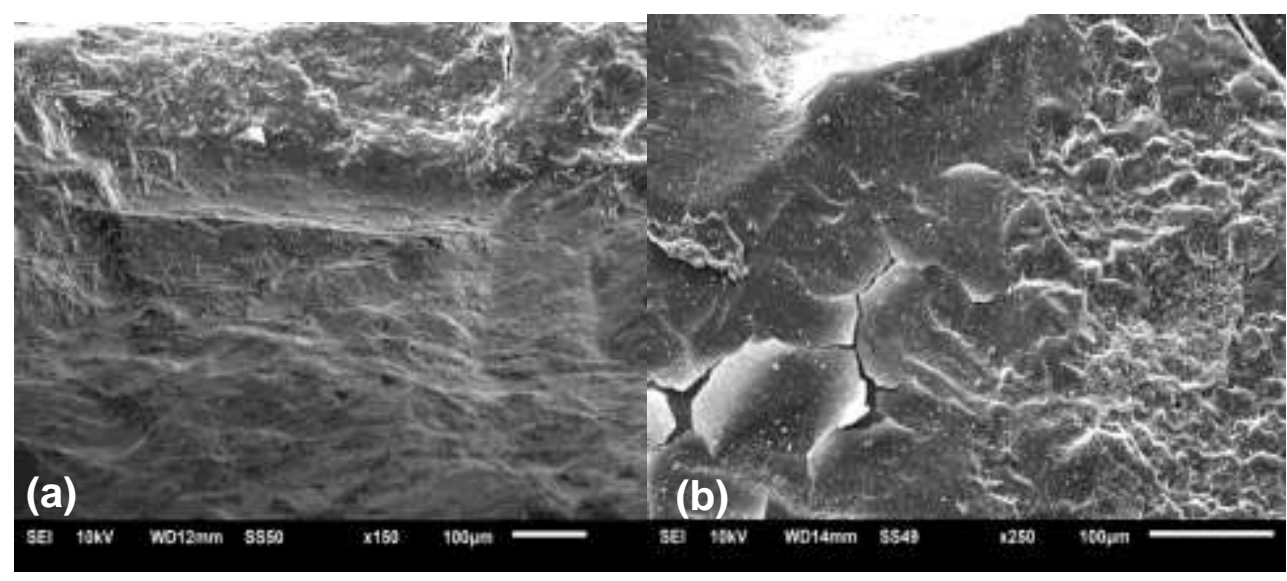

Figura 1 - Imagem de MEV de (a) partícula sem recobrimento e (b) partícula recoberta.

Na Figura 1, pode-se observar as partículas de areia não recobertas (Figura 1a) e as partículas recobertas por quitosana (Figura 1b). A partir destas figuras, nota-se que houve mudança na superfície das partículas após o recobrimento, com a formação de uma película na superfície, mostrando que o recobrimento foi homogêneo em toda a extensão da superfície.

\subsection{Avaliação do Recheio do Leito Frente ao Diâmetro da Partícula}

A Figura 2 apresenta os resultados para os ensaios de adsorção com diferentes tamanhos 
de partículas, onde observa-se que não houve diferença no comportamento dinâmico dos leitos. As curvas de ruptura para ambos os diâmetros foram muito semelhantes na faixa de estudo $(0,4$ a 1,0 mm de diâmetro). Nessa faixa de estudo, os leitos mostraram-se muito permeáveis, assim o efeito do tamanho na partícula no leito não foi observado nessa faixa de estudo.

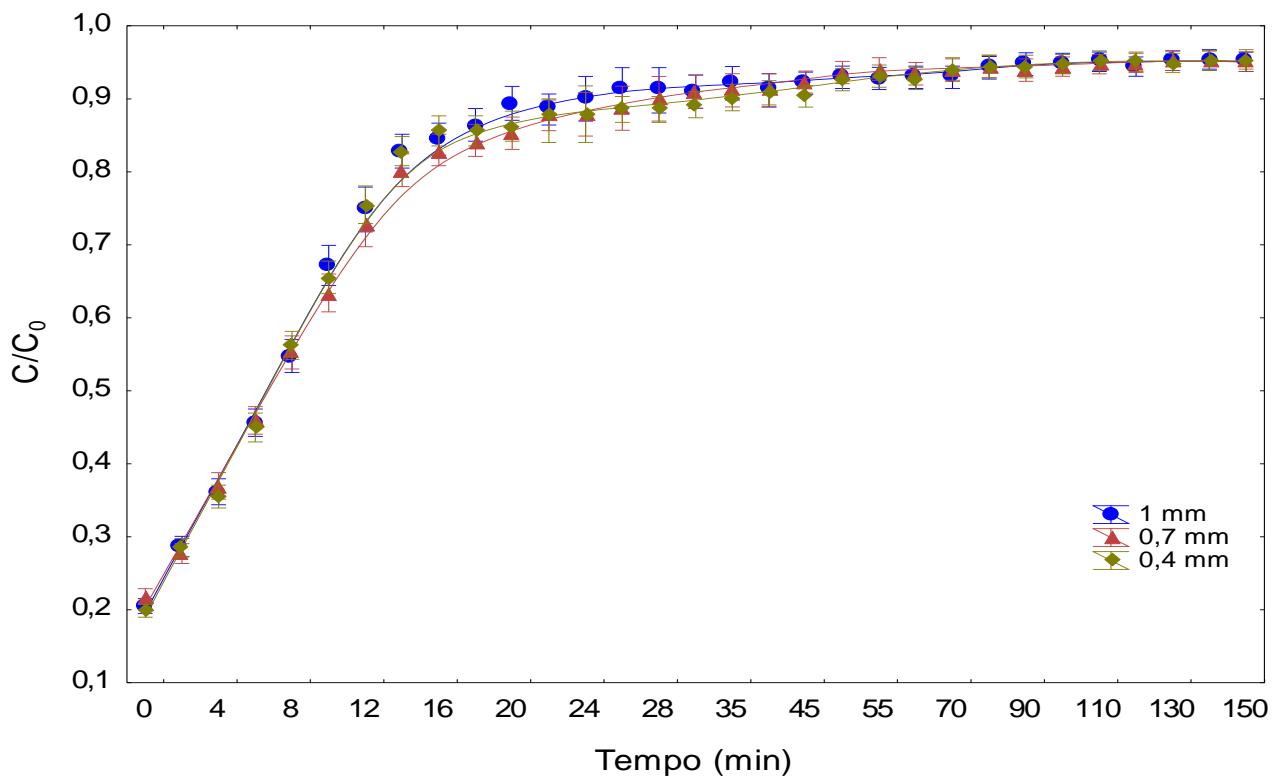

Figura 2 - Avaliação do desempenho na adsorção do cromo (VI) em leito fixo por partículas de areia de diferentes diâmetros recobertas com quitosana.

Tabela 1 - Parâmetros experimentais do leito na avaliação da influência do diâmetro de partícula.

\begin{tabular}{cccc}
\hline $\begin{array}{c}\text { Diâmetro } \\
(\mathrm{mm})\end{array}$ & $q_{t}(\mathrm{mg})$ & $q u(\mathrm{mg} / \mathrm{g})$ & $Z M T(\mathrm{~cm})$ \\
\hline 0,4 & $8,59 \pm 0,25^{\mathrm{a}}$ & $1,63 \pm 0,11^{\mathrm{a}}$ & $23,5 \pm 0,5^{\mathrm{a}}$ \\
0,7 & $8,54 \pm 0,30^{\mathrm{a}}$ & $1,59 \pm 0,17^{\mathrm{a}}$ & $24,5 \pm 0,9^{\mathrm{ab}}$ \\
1,0 & $8,38 \pm 0,20^{\mathrm{a}}$ & $1,62 \pm 0,09^{\mathrm{a}}$ & $24,8 \pm 0,3^{\mathrm{b}}$ \\
\hline
\end{tabular}

*média \pm desvio padrão (n=3). qt: capacidade total de adsorção; qu: capacidade útil;, ZTM: zona de transferência de massa. Letras iguais não apresentam diferença significativa ( $p>0,05)$.

Na Tabela 1 pode-se observar os parâmetros obtidos para o leito fixo com diferentes diâmetros de partículas. Para os três diâmetros de partícula $(0,4 ; 0,7$ e $1.0 \mathrm{~mm})$, os valores de capacidade total de adsorção $\left(q_{t}\right)$, capacidade útil $\left(q_{u}\right)$ não apresentaram diferenças significativas $(\mathrm{p}<0,05)$. Ao analisar a zona de transferência de massa $(Z T M)$, existiu diferença significativa ( $<<0,05)$ nas partículas de diâmetro de $1 \mathrm{~mm}$ quando comparadas com as de 0,4 $\mathrm{mm}$. 


\subsection{Influência da Vazão no Desempenho do Leito}

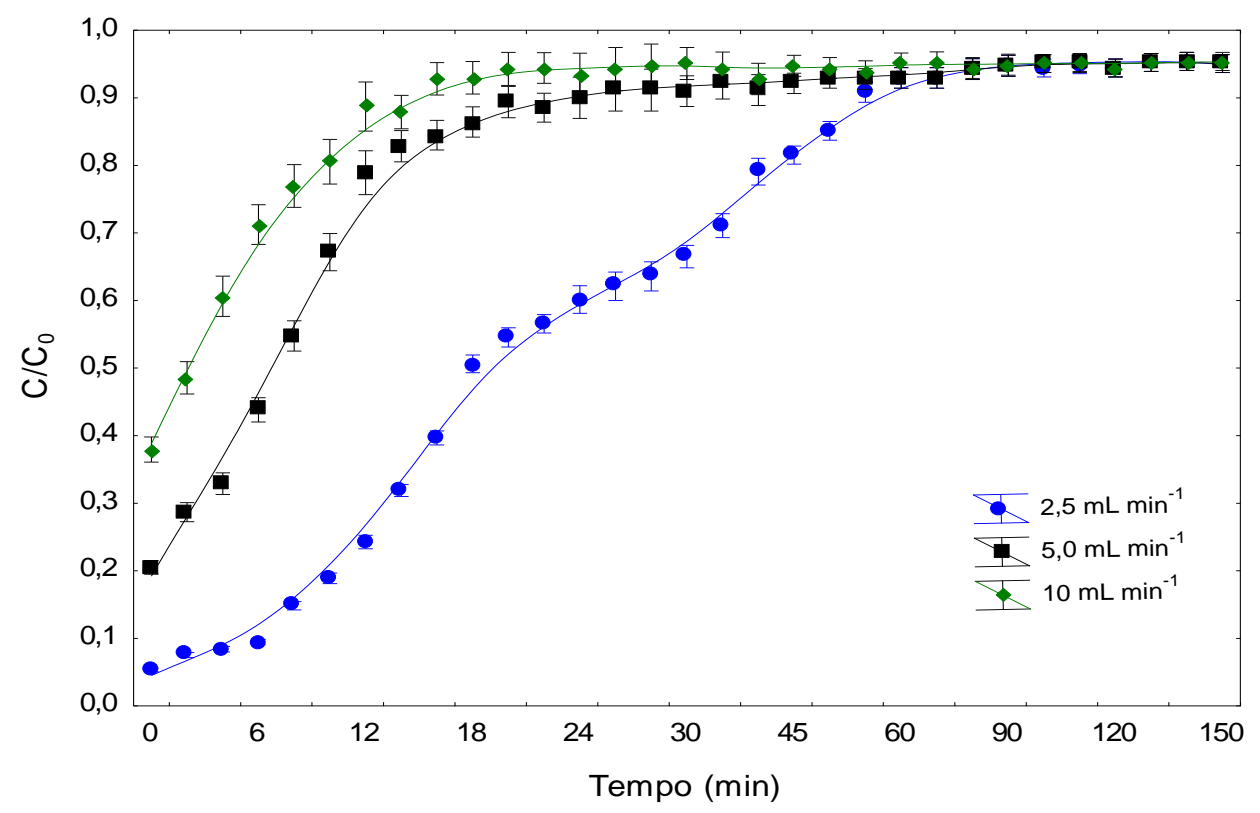

Figura 3 - Influência da vazão da solução na adsorção do cromo (VI) em leito fixo por partículas de areia recobertas com quitosana.

A Figura 3 mostra que na condição de menor vazão, o leito apresentou o melhor desempenho de operação, onde a concentração de saída do efluente foi próxima de zero ou muito baixa. Na condição de maior vazão, o comportamento do leito fixo foi o oposto, sendo a concentração na saída em $t=0$ de $40 \%$ em relação a inicial. Diferente de quando o leito operou em baixa vazão, onde o efluente na saída em $t=0$ estava muito próximo à zero. Hasan et al. (2008) em estudos conduzidos em leito fixo na adsorção de metais obteve comportamento semelhante. Estudos para a adsorção de cromo (VI) utilizando quitosana na forma de pó demonstraram é necessário no mínimo 50 min para se atingir $80 \%$ da saturação (Cadaval et al.,2013).

Na Figura 3 observa-se que na melhor condição de operação $(2,5 \mathrm{~mL} / \mathrm{min})$, o leito apresentou um $T R H$ de $42 \mathrm{~min}$, fazendo com que o leito apresentasse desempenho satisfatório na remoção de cromo (VI) por um curto período. Os resultados sugerem que para a remoção de cromo (VI) para esse sistema de leito fixo proposto, é necessário um tempo de resistência hidráulico $(T R H)$ maior que o observado para a remoção em sistema operando em batelada, devido ao menor tempo de contato entre o adsorvente e adsorbato (Costa e França, 1996).

Na Tabela 2 observa-se que, a redução da vazão em quatro vezes causou um aumento de aproximadamente $150 \%$ na capacidade total do leito e um aumento de aproximadamente $300 \%$ na capacidade útil do leito fixo. 
Tabela 2 - Parâmetros experimentais do leito na avaliação da influência da vazão de alimentação.

\begin{tabular}{cccc}
\hline Vazão $(\mathrm{mL} / \mathrm{min})$ & $q_{t}(\mathrm{mg})$ & $q u(\mathrm{mg} / \mathrm{g})$ & $Z M T(\mathrm{~cm})$ \\
\hline 10,0 & 6,02 & 3,77 & 19,05 \\
5,0 & 8,38 & 7,14 & 16,01 \\
2,5 & 15,60 & 14,85 & 14,67 \\
\hline
\end{tabular}

$q t$ : capacidade total de adsorção; qu: capacidade útil;, ZTM: zona de transferência de massa.

\section{CONCLUSÃO}

Nos ensaios de adsorção no leito fixo, o efeito do tamanho das partículas de areia recobertas com quitosana não mostrou diferença significativa, ao nível de $95 \%$ (p>0,05), no desempenho na adsorção de cromo (IV) em solução aquosa. Na avaliação do efeito da vazão no desempenho do leito na adsorção de cromo (VI), a diminuição da vazão melhorou o desempenho do leito, sendo que as vazões de 5 e $10 \mathrm{~mL} / \mathrm{min}$ não se mostraram adequadas para a operação. A redução da vazão em quatro vezes causou um aumento de aproximadamente $150 \%$ na capacidade total do leito $\left(q_{t}\right)$ e um aumento de aproximadamente $300 \%$ na capacidade útil do leito fixo $\left(q_{u}\right)$.

\section{REFERÊNCIAS}

AlBADARIN, A. B.; MANGWANDI, C.; AL-MUHTASEB, A. H.; WALKER, G. M.; ALLEN, S. J.; AHMAD M.M. Kinetic and thermodynamics of chromium ions adsorption onto low-cost dolomite adsorbent. Chem. Eng. J., v.179, p.193-202, 2012.

AYDIN Y.A.; AKSOY N. D., Adsorption of chromium on chitosan: Optimization, kinetics and thermodynamics. Chem. Eng. J., v.151 p.188-194 2009.

CADAVAL, T. R. S.; CAMARA, A. S.; DOTTO, G. L.; PINTO, L. A. A. Adsorption of Cr (VI) by chitosan with different deacetylation degrees. Desalin. Water Treat. v.51, p.7690-7699, 2013.

COSTA, A. C. A. Y.; FRANÇA, F. P. Biosorption of zinc, cadmium and copper by a brown seaweed (Sargassumsp) in a continuous fixed-bed laboratory reactor. Bioseparation. v.6, p.35-341, 1996.

DOTTO, G. L.; SOUZA, V. C.; PINTO, L. A. A. Drying of chitosan in a spouted bed: The influences of temperature and equipment geometry in powder quality. LWT-Food Sci. Technol., v. 44, p.1786-1792, 2011.

FOUST, A. S.; WENZEL, L. A.; CLUMP, C. W.; MAUS, L.; ANDERSEN, L. B. Principles of Unit Operations. $2^{\text {a }}$ ed. New York: John Wiley \& Sons, 1980.

FUTALAN C.M.; KAN C.C.; DALIDA M.L.; HSIEN K.J.; PASCUA C.; WAN M.-W., Comparative and competitive adsorption of copper, lead, and nickel using chitosan immobilized on bentonite, Carbohyd. Polym., v.83, p.528-536. 2010. 
GEANKOPLIS, C. J. Transport processes and unit operations. $4^{\mathrm{a}}$ ed. New York: PTR Prentice Hall, 1993.

GERENTE, C.; LEE, V. K. C.; CLOIREC, P.P. \& MCKAY, G. Application of chitosan for the removal of metals from wastewaters by adsorption - mechanisms and models review. Crit. Rev. Env. Sci. Tec, v.37, n.1, p.41-127, 2007.

GUIBAL E. Interactions of metal ions with chitosan-based sorbents: a review. Sep. Purif. Technol., v.38, p.43-74, 2004.

HASAN S.; GHOSHA, T. K.; VISWANATH, D. S.; BODDUB, V. M. Dispersion of chitosan on perlite for enhancement of copper (II) adsorption capacity. J. Hazard. Mater., v.152, p.826-837, 2008.

JIANG, X.; CHEN, L.; ZHONG, W.I. A new linear potentiometric titration method for the determination of deacetylation degree of chitosan. Carbohyd. Polym., v.54 p.457-463, 2003.

LAPORTE, R. J. Hidrophilic polymer coatins for medical devices, Structure/Properties, Development, Manufacture and Applications. $4^{a} \mathrm{ed}$., Technomic Publishing Company, Inc, Lancaster Pensylvania, p.169, 1997.

MOURA C. M.; MOURA J. M.; SOARES N. M.; PINTO L. A.A., Evaluation of molar weight and deacetylation degree of chitosan during chitin deacetylation reaction: used to produce biofilm, Chem. Eng. Prog., v.50, p.351-355, 2011.

MUZZARELLI, R. A. A. Colorimetric determination of chitosan. Anal. Biochem., v.260, p.255257, 1998.

OSIFO, P. O.; WEBSTER, A.; VAN DER MERWE, H.; NEOMAGUS, H.W.J.P., VAN DER GUN, M. A.; GRANT, D. M., The influence of the degree of cross-linking on the adsorption properties of chitosan beads. Bioresour. Technol., v.99 p.7377-7382, 2008

VIJAYA, Y.; POPURI, S. R.; BODDU, V. M.; KRISHNAIAH, A. Modified chitosan and calcium alginate biopolymer sorbents for removal of nickel(II) through adsorption. Carbohyd. Polym., v.72, n. 2, p.261-271, 2008.

WANG, H.; LI, C.; BAO, C.; LIU, L.; LIU X., Adsorption and determination of Pd(II) and Pt(IV) onto 30-Nitro-4-amino azobenzene modified chitosan. J. Chem. Eng. Data, v.56, p.4203-4207, 2011.

WESKA, R. F.; MOURA, J. M.; BATISTA, L. M.; RIZZI, J.; PINTO, L. A. A. Optimization of deacetylation in the production of chitosan from shrimp wastes: use of response surface methodology. J. Food Eng., v.80, p.749-753, 2007. 